\title{
Less is More: An Observability Paradox in Repeated Games*
}

\author{
KANDORI, Michihiro ${ }^{\dagger}$ \\ Faculty of Economics \\ University of Tokyo
}

\author{
OBARA, Ichiro \\ Department of Economics \\ $U C L A$
}

August 8, 2006

\begin{abstract}
We present a repeated prisoners' dilemma game with imperfect public monitoring, which exhibits the following paradoxical feature: the (limit) equilibrium payoff set achieves full efficiency asymptotically as the public signal becomes insensitive to the hidden actions of the players. The basic logic behind this result also provides an example where the Folk theorem is obtained, while Fudenberg-Levine-Maskin's sufficient conditions (Econometrica 1994) for Folk theorem are violated.
\end{abstract}

Keywords: Efficiency, Folk Theorem, Garbling, Imperfect Public Monitoring, Prisoners' Dilemma, Repeated Games.

JEL classification codes: C72, C73, D80, D82

Address for correspondence:

Ichiro Obara,

Department of Economics, UCLA

405 Hilgard Ave.

Los Angeles, CA 90095-1477

${ }^{*}$ We would like to thank Drew Fudenberg, David Levine, an associate editor and two anonymous refereees for their helpful comments and suggestions.

${ }^{\dagger}$ e-mail: kandori@e.u-tokyo.ac.jp

${ }^{\ddagger}$ e-mail: iobara@econ.ucla.edu 


\section{Introduction}

Repeated games with imperfect public monitoring provide an analytical framework to study long term relationships, where participants have imperfect public information about each other's hidden action. Now welldeveloped theory (including Abreu, Pearce, and Stacchetti [1] and Fudenberg, Levine, Maskin [7], FLM hereafter) shows how the participants can utilize the imperfect information to achieve cooperation in such a setting. Our intuition suggests that, as the observability of actions improves, it should become easier to sustain cooperation. In fact, Kandori [8] has formalized this idea, by showing that the equilibrium payoff set expands, when observability improves according to the standard definition in statistical decision theory (Blackwell and Girshick [3]). In this paper we point out a subtlety in this issue and show that the above intuitive idea about observability and cooperation should be taken with a pinch of salt. In particular, we present a repeated game with imperfect public monitoring, which has the following seemingly paradoxical feature: the limit PPE payoff set $\left(\lim _{\delta \rightarrow 1} E(\delta)\right)$ can achieve full efficiency asymptotically as the public information becomes less sensitive to the hidden actions of the players. ${ }^{1}$ The basic logic behind this result also provides an example where the Folk theorem is obtained, while FLM's sufficient conditions for the Folk theorem are violated.

Our example is quite simple. We consider the standard prisoners' dilemma stage game with the following payoff table

\begin{tabular}{|c|c|c|}
\hline & $C$ & $D$ \\
\hline$C$ & 1,1 & $-h, 1+d$ \\
\hline$D$ & $1+d,-h$ & 0,0 \\
\hline
\end{tabular}

where $d, h>0$ ( $D$ is dominant) and $d-h<1\left((C, C)\right.$ is efficient $\left.{ }^{2}\right)$. Actions are not observable ${ }^{3}$, but the players publicly observe a signal $\omega \in$

\footnotetext{
${ }^{1}$ Note that less information per se may not be a problem. If the quality of information deteriorates at some action profile, then deviation to the action profile may be more easily detected. A series of paper by E. Lehrer (such as [11],[12]) points out that such distinguishability is the key for supporting more payoffs.

On the other hand, our paper illustrates that more payoffs can be supported as deviations become seemingly less detectable

${ }^{2}$ This is the condition that $(C, C)$ Pareto-dominates the public randomization between $(D, C)$ and $(C, D)$ with an equal probability.

${ }^{3}$ We assume that the realized payoff of a player is a function of his own action and the public signal (so that a player cannot detect the opponent's action simply by looking at his realized payoff), and we denote it by $u_{i}\left(a_{i}, \omega\right)$. The payoff table represents the expected payoff $g_{i}(a)=\sum_{\omega \in \Omega} u_{i}\left(a_{i}, \omega\right) p(\omega \mid a)$, where $p(\omega \mid a)$ is the probability of $\omega$ given
} 
$\Omega \equiv\{X, Y\}$. We suppose that the probability of signal $\omega$ given action profile $a \in\{C, D\} \times\{C, D\}$, denoted $p(\omega \mid a)$, is symmetric (i.e., $p\left(\omega \mid a_{1}, a_{2}\right)=$ $\left.p\left(\omega \mid a_{2}, a_{1}\right)\right)$ and satisfies

$$
\begin{gathered}
p(X \mid C, C)<p(X \mid C, D), \text { and } \\
p(Y \mid D, C)<p(Y \mid D, D) .
\end{gathered}
$$

That is, one defection makes $X$ more likely, while an additional defection makes $Y$ more likely.

We show that this familiar stage game exhibits the following paradoxical feature: As the signal becomes insensitive to a deviation at the efficient point $(C, C)$, (i.e., $p(X \mid C, C)-p(X \mid D, C) \rightarrow 0$, holding $p(X \mid D, D)$ fixed), the limit equilibrium payoff set $\lim _{\delta \rightarrow 1} E(\delta)$ expands and converges to the set of all feasible and individually rational payoffs. (Here we denote the public perfect equilibrium payoff set in the repeated game under discount factor $\delta$ by $E(\delta)$ ). Moreover, this can be true even if observability is reduced everywhere (not just at the efficient point): as $p(\omega \mid a)$ for all $\omega$ and $a$ converges to one point (so that a deviation at any point becomes hard to detect), the asymptotic payoff set expands and converges to the set of all feasible and individually rational payoffs as long as $p(X \mid C, C)-p(X \mid$ $D, C)$ converges to 0 faster than $p(X \mid D, C)-p(X \mid D, D)$.

The mechanism operating behind our example is a familiar one (at least to the specialists in repeated games). Our point is to show that the familiar mechanism can operate in a subtle and disguised way. For this reason (and just for fun) we pause here for a moment and ask the reader if $\mathrm{s} /$ he can see how our example works. The answer is given in the next section and we show how the trick is done.

\section{Intuitive Explanation}

The key for our result is the efficiency of asymmetric punishment. When monitoring is imperfect, a "bad" outcome arises with a positive probability even if no player deviates. To provide incentives to cooperate, a punishment should be triggered in such an eventuality. If all players are simultaneously punished (as in the trigger strategy), welfare loss is inevitable. However, when each player's action affects the signal asymmetrically, we can use an

action profile $a$. Throughout the paper we hold the expected payoff fixed when we change the information structure $p(\omega \mid a)$. One can check that such an operation is possible with a suitable choice of $u_{i}\left(a_{i}, \omega\right)$. 
asymmetric punishment scheme to avoid welfare loss; if player 1's deviation is suspected, we transfer 1's future payoff to player 2 (and vice versa). When the discount factor $\delta$ is close to 1 , such a transfer can be made in a close vicinity of the Pareto frontier, and therefore the welfare loss vanishes as $\delta \rightarrow 1$. Furthermore, this conclusion is independent of how informative the signal is (as long as each player's deviation affects the signal asymmetrically). This is the basic driving force in the FLM folk theorem [7].

In our example, each player's action affects the signal symmetrically at the efficient point $(C, C)$ (each player's deviation makes $X$ more likely), so that we are unable to use the efficient asymmetric punishment at this point. Hence, the above argument seemingly implies that efficiency cannot be achieved. However, consider a nearby point $\left(D_{\varepsilon}, C\right)$, where $D_{\varepsilon}$ denotes the mixed strategy which plays $C$ and $D$ with probabilities $(1-\varepsilon)$ and $\varepsilon>0$ respectively. Player 1's deviation to $D$ makes $X$ more likely at this point, as at $(C, C)$. What about player 2's defection? The distribution of signal when player 2 takes action $a_{2}$ is given by

$$
(1-\varepsilon) p\left(\omega \mid C, a_{2}\right)+\varepsilon p\left(\omega \mid D, a_{2}\right) .
$$

Now suppose that the first term is sufficiently insensitive to $a_{2}$. Then, player 2's action affects the signal mostly through the second term. As $p(Y \mid D, C)<p(Y \mid D, D)$, we conclude that player 2's defection makes $Y$ more likely at this point. Hence, each player's action affects the signal asymmetrically at $\left(D_{\varepsilon}, C\right)$, so that the efficient asymmetric punishment is feasible. ${ }^{4,5}$ Note that $\varepsilon$ can be made arbitrarily small as the signal becomes insensitive at the efficient point $(C, C)$. Hence, as observability decreases, the asymmetric punishment becomes feasible at almost efficient point $\left(D_{\varepsilon}, C\right)$ (and symmetrically at $\left.\left(C, D_{\varepsilon}\right)\right)$. This is the source of efficiency in our examples. Hence, the crux of the matter is the feasibility of asymmetric punishment, not the amount of information per se. The twist of our example is that the former is attained in a neighborhood of the efficient action profile $(C, C)$ only when the observability of actions is decreased (in a broad sense).

Now let us make the above argument more precise. At $\left(D_{\varepsilon}, C\right)$, the

\footnotetext{
${ }^{4} \mathrm{~A}$ basic idea behind this construction is that a monitoring structure can be improved by utilizing an inefficient action profile. This general idea is also the main theme of our related paper [10].

${ }^{5}$ Lemma 6.3 in Fudneberg, Levine, and Maskin [7] also employs a mixed action to sustain an efficient outcome which may not be otherwise sustainable. Mixed strategies also play an important role in the literature of repeated games with private monitoring such as Sekiguchi [14], Bhaskar and Obara [2], Piccione [13], and Ely and Välimäki [5].
} 
change in the probability of $Y$ when player 2 deviates is equal to

$$
(1-\varepsilon)[p(Y \mid C, D)-p(Y \mid C, C)]+\varepsilon[p(Y \mid D, D)-p(Y \mid D, C)] .
$$

If this expression is positive, player 2's defection at $\left(D_{\varepsilon}, C\right)$ makes $Y$ more likely, so that each player's action affects the signal asymmetrically, as we desire. The above expression (1) can be rearranged as

$$
-(1-\varepsilon) \xi+\varepsilon \Delta
$$

where $\xi \equiv p(X \mid C, D)-p(X \mid C, C)>0$ and $\Delta \equiv p(Y \mid D, D)-p(Y \mid D, C)>0$. Hence, (1) is positive if and only if

$$
\varepsilon>\frac{\xi}{\xi+\Delta}
$$

Given $\xi>0$ and $\Delta>0$, this inequality provides a lower bound on the probability of $D$. Hence $\left(D_{\varepsilon}, C\right)$ is bounded away from the efficient action profile $(C, C)$ when the efficient asymmetric punishment is employed. However, this lower bound vanishes when the observability of a deviation at $(C, C)$ is reduced (i.e., $\xi \rightarrow 0$ ). Furthermore, the same is true even when the observability of action is reduced everywhere (i.e., $\xi \rightarrow 0$ and $\Delta \rightarrow 0$ ) as long as $\Delta$ converges to 0 at a slower rate than $\xi$. Thus we can employ an asymmetric punishment which approximates the efficient action profile $(C, C)$ arbitrarily closely as $\xi \rightarrow 0$ for such $\Delta .{ }^{6}$

Although this is the essence of our argument, we should address other non-trivial issues to obtain the formal proof. First, when supporting ( 1 $\varepsilon) C+\varepsilon D, C)$, player 1 must be indifferent between $C$ and $D$. This indifference condition determines the level of transfer between 1 and 2 in the efficient asymmetric punishment. In general, this transfer may not be sufficient to deter player 2's deviation. We will show ${ }^{7}$ that this does not happen when $\xi \rightarrow 0$ and $\xi / \Delta \rightarrow 0$. Second, we need to show that the continuation payoffs in the asymmetric punishment are also sustained by the repeated game equilibria. The detailed analysis is given in the next section.

Lastly, let us comment on the relationship of our result to the folk theorem under imperfect public monitoring (FLM [7]). Our example does not satisfy the FLM conditions for the folk theorem for each $\xi>0$ and neither

\footnotetext{
${ }^{6}$ On the other hand, if $\xi / \triangle$ is bounded away from 0 , then the public signal distribution always moves in the same direction whoever is deviating to $D$ in a small enough neighborhood of $(C, C)$. This implies that asymmetric punishments cannot be used around $(C, C)$, hence the efficient outcome cannot be approximated by any PPE in this case.

${ }^{7}$ A detailed verbal explanation is provided in Case 3 of the proof of Lemma 2.
} 
in the limit $\xi \rightarrow 0$. Their pairwise full rank condition requires that there is at least one (potentially mixed) action profile $\alpha$ for which matrix

$$
\left(\begin{array}{cc}
p\left(X \mid C, \alpha_{2}\right) & p\left(Y \mid C, \alpha_{2}\right) \\
p\left(X \mid D, \alpha_{2}\right) & p\left(Y \mid D, \alpha_{2}\right) \\
p\left(X \mid \alpha_{1}, C\right) & p\left(Y \mid \alpha_{1}, C\right) \\
p\left(X \mid \alpha_{1}, D\right) & p\left(Y \mid \alpha_{1}, D\right)
\end{array}\right)
$$

has rank 3. Obviously this is impossible, because this matrix has only two columns. However, our example is based on the fundamental idea of FLM, the efficiency of asymmetric punishment. The point here is that the pairwise full rank condition is a sufficient condition to facilitate the efficient asymmetric punishment, but it is not necessary. The essence of our example is that (i) the efficient asymmetric punishment is feasible at some points, even though the pairwise full rank condition fails, and (ii) those points can be arbitrarily close to the efficient point, as the observability is reduced. This suggests that we may obtain the folk theorem without the pairwise full rank condition. In fact, we show in Section 5 that we can modify this model to obtain a fairly simple example which satisfies the folk theorem without the pairwise full rank condition.

We present the formal statement of our result and its proof in the next section and generalize it to a more general class of stage games. Then we offer some discussion on its implication on the the folk theorem in Section 5 and Kandori [8] in the last section.

\section{Analysis}

We denote player $i^{\prime}$ s expected payoff given $a \in A=A_{1} \times A_{2}$ by $g_{i}(a)$, and, by abusing notation, $g_{i}(\alpha)$ for player $i^{\prime}$ s expected payoff given a mixed action profile $\alpha \in \triangle A_{1} \times \triangle A_{2}$. Let $V^{*}$ be the individually rational and feasible set, i.e. $V^{*}=\{v \in \operatorname{Cog}(A) \mid v \geq 0\}$, where $\operatorname{Cog}(A)$ denotes the convex hull of set $g(A)=\{v \mid v=g(a), a \in A\}$. Note that in the prisoners' dilemma game we consider, 0 is the minimax payoff for each player.

We use the Fudenberg and Levine's algorithm to compute the asymptotic (perfect public) equilibrium payoff set ([6]). Let us briefly summarize their method for readers' convenience. For a given welfare weight vector $\lambda \in$ $\Re^{2} \backslash\{\mathbf{0}\}$ and a mixed action profile $\alpha$, let $k^{*}(\alpha, \lambda)$ be the maximized value for the following static optimization problem ${ }^{8}$.

\footnotetext{
${ }^{8}$ This is a bit different from their exposition. We used the fact that $v=(1-\delta) g(\alpha)+$
} 


$$
\begin{aligned}
& \max _{v_{i}, x_{i}(\cdot), i=1,2} \lambda \cdot(g(\alpha)+E[x(\omega) \mid \alpha]) \text { subject to } \\
& \forall i \forall a_{i} g_{i}(\alpha)+E\left[x_{i}(\omega) \mid \alpha\right] \geq g_{i}\left(a_{i}, \alpha_{-i}\right)+E\left[x_{i}(\omega) \mid a_{i}, \alpha_{-i}\right] \\
& \text { and the equality holds for } a_{i} \in \operatorname{supp} \alpha_{i} \\
& \forall \omega \lambda \cdot x(\omega) \leq 0
\end{aligned}
$$

where $\operatorname{supp} \alpha_{i}$ is the support of $\alpha_{i}$. Roughly speaking, the term $x$ in the above optimization problem represents the variation in continuation payoffs, and the constraint $\lambda \cdot x(\omega) \leq 0$ ensures that the payoff variations lie in the equilibrium payoff set. Let $k^{*}(\lambda)=\sup _{\alpha} k^{*}(\alpha, \lambda)$ and $H(\lambda)$ be the half-space given by $\left\{v \in \Re^{2} \mid \lambda \cdot v \leq k^{*}(\lambda)\right\}$. Let $Q=\cap_{\lambda \in \Re^{2} \backslash\{\mathbf{0}\}} H(\lambda)$, and denote the set of perfect public equilibrium ${ }^{9}$ payoffs for discount factor $\delta$ by $E(\delta)$. Then Fudenberg and Levine [6] proved the following:

F-L Algorithm: $Q=\lim _{\delta \rightarrow 1} E(\delta)$ when $Q$ has an interior point.

Let us parametrize the information structure by

$$
\begin{aligned}
\xi & \equiv p(X \mid C, D)-p(X \mid C, C)>0, \text { and } \\
\Delta(\xi) & \equiv p(Y \mid D, D)-p(Y \mid D, C)>0
\end{aligned}
$$

while $p(\omega \mid C, C)$ is fixed. To indicate explicitly the dependence of $Q$ in the above result on the parameter $\xi$, we denote it by $Q(\xi)$. We examine how $Q(\xi)$ is affected by $\xi$. For each $\xi$, we can show that $Q(\xi)$ is a strict subset of $V^{*}$. In particular, it is bounded away from the efficient payoff profile $(1,1)$. However, we show that $Q(\xi)$ expands and supports almost all individually rational payoff profile as $\xi$ converges to 0 as long as $\Delta(\xi)$ is bounded away from 0 or converges to 0 at a slower rate. Therefore full efficiency is achieved only in the limit as $\xi \rightarrow 0$. One example of such information structure would be $p(X \mid C, C)=1 / 2$ and $\Delta(\xi)=\xi+1 / 4$ (so that $p(Y \mid D, D)=3 / 4$ is constant). Another example would be $p(X$

$\overline{\delta E[u(\omega) \mid \alpha] \text { is equivalent to } v=g(\alpha)}+E[x(\omega) \mid \alpha]$ for $x(\omega)=\frac{\delta}{1-\delta}(u(\omega)-v)$ (the former is obtained by the latter multiplied by $(1-\delta))$.

${ }^{9}$ Perfect public equilibrium is sequential equilibrium where each player's action depends only on the history of publicly observable signals. 
| $C, C)=1 / 2$ and $\Delta(\xi)=\sqrt{\xi}$ (so that $p(\omega \mid a) \rightarrow 1 / 2$ for all $\omega$ and $a$, as $\xi \rightarrow 0)$. Now we prove the following result. Recall that $E(\delta)$ is the set of perfect public equilibrium payoffs under discount factor $\delta$. Note also that, for any $\delta^{\prime} \in(0,1), E\left(\delta^{\prime}\right)$ is contained in $\lim _{\delta \rightarrow 1} E(\delta) .{ }^{10}$

Theorem 1 For each $\xi>0$, the equilibrium payoffs are bounded away from the efficient point $(1,1)$; there is a neighborhood of $(1,1)$ which lies outside of $\lim _{\delta \rightarrow 1} E(\delta)$. However, as the signal becomes less sensitive to actions ( $\xi \rightarrow$ $0)$, the inefficiency vanishes and we can sustain all feasible and individually rational payoffs $\left(\lim _{\delta \rightarrow 1} E(\delta) \rightarrow V^{*}\right.$ as $\left.\xi \rightarrow 0\right)$, when $\lim _{\xi \rightarrow 0} \frac{\xi}{\Delta(\xi)}=0 .{ }^{1112}$

We need to mention that, although $\lim _{\delta \rightarrow 1} E(\delta)$ converges to $V^{*}$ as $\xi \rightarrow$ 0 , we do not know whether it expands to $V^{*}$ monotonically as $\xi \rightarrow 0$.

The proof of this theorem is given by the Fudenberg-Levine Algorithm and the following Lemmata.

Lemma $1(1,1) \notin Q(\xi)$ for any $\xi>0$.

Lemma $2 \lim _{\xi \rightarrow 0} Q(\xi)=V^{*}$ if $\lim _{\xi \rightarrow 0} \frac{\xi}{\Delta(\xi)}=0$.

Note that Lemma 1 immediately implies the first part of Theorem 1, because $Q(\xi)\left(=\lim _{\delta \rightarrow 1} E(\delta)\right)$ is closed (as it is an intersection of closed half-spaces). The proof of Lemma 1 is conceptually quite simple. To sustain a point near $(1,1)$, the players need to play $C$ with a sufficiently large probability, which implies that each player's defection makes $X$ more likely. This means that both players should be punished when $X$ arises. As this entails welfare loss, it is impossible to sustain any point near $(1,1)$. This intuitive argument can be formalized as follows.

\footnotetext{
${ }^{10}$ See Fudenberg and Levine $([6])$, Theorem 3.1 .

${ }^{11}$ Thus the order of the limits matters. For example, take a sequence of $\xi$ such that $\xi \rightarrow 0, \Delta(\xi) \rightarrow 0$ and $\lim _{\xi \rightarrow 0} \frac{\xi}{\Delta(\xi)}=0$. We have $\lim _{\xi \rightarrow 0} \lim _{\delta \rightarrow 1} E(\delta)=V^{*}$, while $\lim _{\delta \rightarrow 1} \lim _{\xi \rightarrow 0} E(\delta)$ is $(0,0)$ because $E(\delta)=(0,0)$ at $\xi=0$ for any $\delta$.

${ }^{12}$ This theorem also implies that efficiency may be improved as information gets worse for a given discount factor. More precisely, we can find $\xi$ and $\xi^{\prime}(<\xi)$ satisfying $\frac{\xi^{\prime}}{\Delta\left(\xi^{\prime}\right)}<$ $\frac{\xi}{\Delta(\xi)}$ and $\underline{\delta}$ such that, for any fixed $\delta \in(\underline{\delta}, 1)$, a PPE payoff with $\xi^{\prime}$ is closer to $(1,1)$ than any $\mathrm{PPE}$ payoff with $\xi$.
} 
Proof of Lemma 1: It is sufficient to demonstrate $(1,1) \notin H((1,1))$. Recall that this is equivalent to

$$
\begin{aligned}
(1,1) \cdot(1,1) & >\sup \{(1,1) \cdot(g(\alpha)+E[x(\omega) \mid \alpha])\} \\
2 & >\sup \left\{\sum_{i=1}^{2} g_{i}(\alpha)+E\left[x_{i}(\omega) \mid \alpha\right]\right\}
\end{aligned}
$$

where the supremum is taken over $\alpha$ and $x$ such that $\alpha$ is enforced by $x$ which satisfies $x_{1}(\omega)+x_{2}(\omega) \leq 0$ for all $\omega$. Recall that $D_{\varepsilon}$ denotes the behavior strategy which plays $D$ with probability $\varepsilon$. Let $\varepsilon^{\prime \prime}$ be the minimum $\varepsilon$ such that $p\left(X \mid D_{\varepsilon}, D\right) \leq p\left(X \mid D_{\varepsilon}, C\right)$, and let $M \equiv\left\{\alpha \mid \alpha_{i}(D) \geq \varepsilon^{\prime \prime}(\xi)\right.$ for at least one $i=1,2\}$. Note that $\varepsilon^{\prime \prime}(\xi)=\frac{\xi}{\xi+\Delta(\xi)}>0$ by assumption. Then there exists some $K>0$ such that for any $\alpha \in M$

$$
\begin{aligned}
2-K & >\sum_{i=1}^{2} g_{i}(\alpha) \\
& \geq \sum_{i=1}^{2} g_{i}(\alpha)+E\left[x_{i}(\omega) \mid \alpha\right]
\end{aligned}
$$

as $\sum_{i=1}^{2} x_{i}(\omega) \leq 0$ for all $\omega$. Hence, (2) is proved if we show that there is another constant $K^{\prime}>0$ such that for all $\alpha \notin M$

$$
2-K^{\prime} \geq \sum_{i=1}^{2} g_{i}(\alpha)+E\left[x_{i}(\omega) \mid \alpha\right] .
$$

Note that, when $\alpha \notin M$, each player plays $D$ with probability less than $\varepsilon^{\prime \prime}(\xi)$. This means that, by the definition of $\varepsilon^{\prime \prime}(\xi)$, any player's deviation makes $X$ more likely, so that both players should be punished when $X$ is realized. Player 1's incentive constraint is

$$
d \leq\left\{p\left(X \mid D, \alpha_{2}\right)-p\left(X \mid C, \alpha_{2}\right)\right\}\left(x_{1}(Y)-x_{1}(X)\right),
$$

and by using this we can obtain an upper bound for $g_{1}(\alpha)+E\left[x_{1}(\omega) \mid \alpha\right]$ :

$$
\begin{aligned}
& g_{1}\left(C, \alpha_{2}\right)+E\left[x_{1}(\omega) \mid C, \alpha_{2}\right] \\
= & g_{1}\left(C, \alpha_{2}\right)+x_{1}(Y)-p\left(X \mid C, \alpha_{2}\right)\left(x_{1}(Y)-x_{1}(X)\right) \\
\leq & g_{1}\left(C, \alpha_{2}\right)+x_{1}(Y)-\frac{d}{L^{\alpha_{2}}-1}
\end{aligned}
$$


where $L^{\alpha_{2}}=\frac{p\left(X \mid D, \alpha_{2}\right)}{p\left(X \mid C, \alpha_{2}\right)}>1$ is the likelihood ratio given $\alpha_{2}$. Similarly, we can obtain $g_{2}\left(\alpha_{1}, C\right)+x_{2}(Y)-\frac{d}{L^{\alpha_{1}-1}}$ as an upper bound for $g_{2}(\alpha)+E\left[x_{2}(\omega) \mid \alpha\right]$. These inequalities, together with the constraint $\sum_{i=1}^{2} x_{i}(Y) \leq 0$, imply

$$
\begin{aligned}
& \sum_{i=1}^{2} g_{i}(\alpha)+E\left[x_{i}(\omega) \mid \alpha\right] \\
\leq & \left(g_{1}\left(C, \alpha_{2}\right)-\frac{d}{L^{\alpha_{2}}-1}\right)+\left(g_{2}\left(\alpha_{1}, C\right)-\frac{d}{L^{\alpha_{1}}-1}\right) \\
\leq & 2-\frac{2 d}{L-1}
\end{aligned}
$$

for any $\alpha \notin M$ and $x$, where $L \equiv \frac{p(X \mid D, C)}{p(X \mid C, C)}$. Q.E.D.

Proof of Lemma 2: To establish the claim we will identify certain point(s) contained in the half-space $H(\lambda)$ for each direction $\lambda \neq 0$. A convex-hull of such points provides a subset of $Q(\xi)$, and we will show that this subset tends to $V^{*}$ as $\xi \rightarrow 0$.

Case 1, $\lambda_{1}, \lambda_{2} \leq 0$ and $\lambda \neq 0:(0,0) \in H(\lambda)$.

This is true because Nash equilibrium $(0,0)$ is sustained by $x_{i} \equiv 0$, $i=1,2$. Note that the above claim implies $\cap_{\lambda_{1}, \lambda_{2} \leq 0, \lambda \neq 0} H(\lambda) \supset \Re_{+}^{2} \equiv$ $\left\{v \in \Re^{2} \mid v_{i} \geq 0\right\}$.

Case 2, $\lambda_{1}>0, \lambda_{2} \leq 0: g(D, C) \in H(\lambda)$.

This is established by showing that $(D, C)$ is enforceable while $\lambda \cdot x(\omega)=$ 0 is satisfied. This is an easy case, because any player's deviation at this point makes $Y$ more likely, and $\lambda_{1}>0, \lambda_{2} \leq 0$ implies that the sidepayment scheme $x$ can punish both players when $Y$ arises without any welfare $\operatorname{loss}^{13}$ (i.e., $\lambda \cdot x(\omega)=0$ ). The incentive constraints are

$$
\begin{aligned}
& g_{1}(D, C)+E\left[x_{1}(\omega) \mid D, C\right] \geq g_{1}(C, C)+E\left[x_{1}(\omega) \mid C, C\right] \\
& g_{2}(D, C)+E\left[x_{2}(\omega) \mid D, C\right] \geq g_{2}(D, D)+E\left[x_{2}(\omega) \mid D, D\right],
\end{aligned}
$$

\footnotetext{
${ }^{13}$ Precisely speaking, when $\lambda_{2}=0$, the efficient punishment condition $\lambda \cdot x \equiv 0$ requires that player 1 receives no punishment $\left(x_{1} \equiv 0\right)$. However, this is not a problem, as player 1 is taking a myopic best reply at $(D, C)$.
} 
which reduce to

$$
\begin{aligned}
& d \geq \xi\left(x_{1}(Y)-x_{1}(X)\right) \\
& h \leq \Delta(\xi)\left(x_{2}(X)-x_{2}(Y)\right) .
\end{aligned}
$$

First, $\lambda \cdot x(\omega)=0$ can be satisfied by setting $x_{1}(Y)=x_{2}(Y)=0, x_{1}(X)=$ $-\frac{\lambda_{2}}{\lambda_{1}} x_{2}(X)$. The incentive constraints are satisfied by taking $x_{2}(X)$ large enough, because $-\frac{\lambda_{2}}{\lambda_{1}}$ is non-negative. Therefore $(D, C)$ is achieved without any efficiency loss, which implies $g(D, C) \in H(\lambda)$.

Recall that $D_{\varepsilon}$ denotes a mixed action profile where $D$ is played with probability $\varepsilon$. With this notation, we show the following.

Case $3^{14}, \lambda_{1} \geq \lambda_{2}>0$ : For a fixed $\varepsilon \in(0,1)$, we have

$$
g\left(D_{\varepsilon^{\prime}}, C\right) \in H(\lambda)
$$

for all $\varepsilon^{\prime} \in[\varepsilon, 1]$ if $\frac{\xi}{\Delta(\xi)}$ is sufficiently small.

Before going into the formal proof, let us provide some intuition. We first discuss the case $\varepsilon^{\prime} \neq 1$ so that player 1 is mixing $C$ and $D$. Calculation shows that, if $\varepsilon^{\prime}>\frac{\xi}{\xi+\Delta(\xi)}$, the profitable deviation (to $D$ ) by each player affects the signal asymmetrically. That is, defection by 1 makes $X$ more likely, while defection by 2 makes $Y$ more likely. Hence, to deter the profitable deviations, we may transfer payoff from 2 to 1 when $Y$ arises and vice versa. This causes no welfare loss $(\lambda \cdot x(\omega)=0)$.

However, this is not the end of the story, because 1 should be indifferent between $C$ and $D$. This indifference condition determines the magnitude of the transfer $x$, and it should be sufficiently large to deter player 2's defection. Given such limited resource for punishment for player 2, the signal must not be too insensitive to 2's defection. Since the signal becomes completely insensitive to 2's action when the mixing probability $\varepsilon^{\prime}$ is exactly equal to $\frac{\xi}{\xi+\Delta(\xi)}, \varepsilon^{\prime}$ must be larger than $\frac{\xi}{\xi+\Delta(\xi)}$ by some bounded amount. If $\frac{\xi}{\Delta(\xi)}$ is small enough, we can guarantee that such condition is satisfied for any $\varepsilon^{\prime} \in[\varepsilon, 1]$.

Finally, the case $\varepsilon^{\prime}=1$ can be treated as a special case of the above construction (although a much simpler argument works, because player 1

\footnotetext{
${ }^{14}$ Symmetric arguments apply to the remaining case: $\lambda_{2} \geq \lambda_{1}>0$.
} 
need not be indifferent between $C$ and $D$ in this case). This completes the intuitive explanation, and now let us provide the formal proof for Case 3.

The incentive constraints are

$$
\begin{aligned}
d & =\xi\left(x_{1}(Y)-x_{1}(X)\right)(1 \text { is indifferent }), \text { and } \\
\varepsilon h+(1-\varepsilon) d & \leq\left\{p\left(Y \mid D_{\varepsilon}, D\right)-p\left(Y \mid D_{\varepsilon}, C\right)\right\}\left(x_{2}(X)-x_{2}(Y)\right) \\
& =\{-(1-\varepsilon) \xi+\varepsilon \Delta(\xi)\}\left(x_{2}(X)-x_{2}(Y)\right)(2 \text { does not want to play } D) .
\end{aligned}
$$

These constraints and $\lambda \cdot x(\omega)=0$ for $\omega \in(X, Y)$ are satisfied, if we choose $x_{1}(X)=0, x_{1}(Y)=\frac{d}{\xi}, x_{2}(X)=0$, and $x_{2}(Y)=-\frac{\lambda_{1}}{\lambda_{2}} \frac{d}{\xi}$. As for the second constraint, the RHS satisfies

$$
\begin{aligned}
& \{-(1-\varepsilon) \xi+\varepsilon \Delta(\xi)\}\left(x_{2}(X)-x_{2}(Y)\right) \\
= & \{\varepsilon(\xi+\Delta(\xi))-\xi\} \frac{\lambda_{1}}{\lambda_{2}} \frac{d}{\xi} \\
\geq & \left.d\left\{\varepsilon \frac{\xi+\Delta(\xi)}{\xi}-1\right\} \text { (because } \lambda_{1} \geq \lambda_{2}>0\right) .
\end{aligned}
$$

Since $\frac{\xi+\Delta(\xi)}{\xi}$ blows up as $\frac{\xi}{\Delta(\xi)} \rightarrow 0$, the second inequality is also satisfied if $\frac{\xi}{\Delta(\xi)}$ is small enough. ${ }^{15}$ Hence, for given $\varepsilon>0, g\left(D_{\varepsilon^{\prime}}, C\right) \in H(\lambda)$ holds for any $\varepsilon^{\prime} \in[\varepsilon, 1]$ as $\frac{\xi}{\Delta(\xi)} \rightarrow 0$.

Our analysis of Case 3 makes it clear that, for any sequence of $\xi \rightarrow 0$ such that $\frac{\xi}{\Delta(\xi)} \rightarrow 0$, we can find a sequence $\varepsilon^{\prime}(\xi) \in(0,1)$ such that $\varepsilon^{\prime}(\xi) \rightarrow 0$ and player 2's incentive constraint is satisfied, hence $g\left(D_{\mathcal{\varepsilon}^{\prime}(\xi)}, C\right) \in H(\lambda)$ for each $\varepsilon^{\prime}(\xi)$. Then $Q(\xi)$ contains

$$
\underline{Q}(\xi) \equiv \Re_{+}^{2} \cap \operatorname{Co}\left\{g\left(D_{\varepsilon^{\prime}(\xi)}, C\right), g\left(C, D_{\varepsilon^{\prime}(\xi)}\right), g(D, C), g(C, D),(0,0)\right\},
$$

where Co denotes the convex hull (see Figure A). The reader can verify from the figure that $\underline{Q}(\xi) \subset H(\lambda)$ for each case discussed above (Case 1 is depicted in the figure).

\footnotetext{
${ }^{15}$ Calculation shows that $d\left\{\varepsilon \frac{\xi+\Delta(\xi)}{\xi}-1\right\}$ is larger than $\varepsilon h+(1-\varepsilon) d$ if $\frac{\xi}{\Delta(\xi)}$ is smaller than

$$
\frac{1}{\frac{h}{d}+\frac{2}{\varepsilon}-2}
$$
}




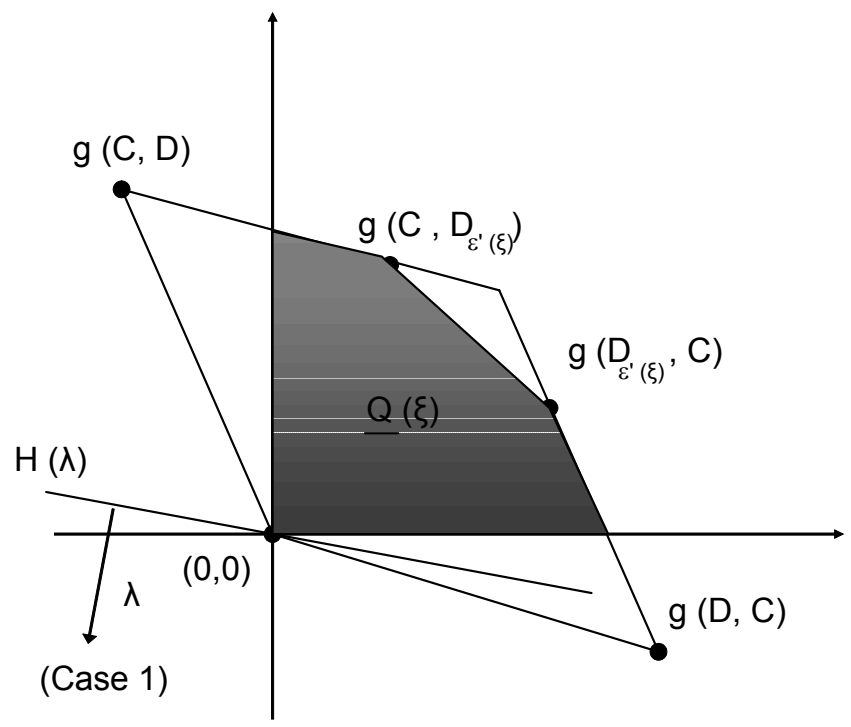

Figure A

Note that $Q(\xi) \rightarrow V^{*}$ as $\xi \rightarrow 0$, because $\varepsilon^{\prime}(\xi) \rightarrow 0$. This and the fact $Q(\xi)=\lim _{\delta \rightarrow 1} \bar{E}(\delta) \subset V^{*}$ prove Lemma 2. Q.E.D.

\section{A Generalization}

The core of our example is that an action arbitrarily close to the efficient point can be supported by transfers of continuation payoffs across players, when the signal becomes uninformative at the efficient point. Now we show that a similar construction is possible for a class of games which are more general than the prisoners' dilemma.

Our generalized model has many actions and many signal outcomes. The stage game is symmetric and has a symmetric efficient point $\left(a^{1}, a^{1}\right)$, which is not a Nash equilibrium. By symmetry, each player's deviation at this point affects the signal in a symmetric way, so there is no way to enforce this outcome by transfer of payoffs across players (i.e. the only way to enforce the efficient action profile is to punish the players simultaneously, when a "bad" outcome arises.). However, a point close to the efficient point, $\left((1-\varepsilon) \circ a^{1}+\varepsilon \circ a^{m}, a^{1}\right)$, where player 1 is mixing some action $a^{m}$ with a small probability, is shown to be enforced by transfer, when the signal is 
sufficiently insensitive at the efficient point. ${ }^{16,17}$

The public signal takes on $H$ different values $\omega \in \Omega=\left\{\omega^{1}, \ldots, \omega^{H}\right\}$. As in our previous example, we assume that the signal space is too small for the FLM folk theorem to hold. Recall that the pairwise full rank condition of FLM is satisfied only if $|\Omega| \geq\left|A_{1}\right|+\left|A_{2}\right|-1$, or in our notation, $H \geq 2 K-1$. Hence we assume $H<2 K-1$. On the other hand, we assume that the number of signal outcomes are not too small, so that the individual full rank condition is satisfied (at the efficient point). To state this assumption, consider a row vector of signal distribution given action profile $a$,

$$
p(a) \equiv\left(p\left(\omega^{1} \mid a\right), \ldots, p\left(\omega^{H} \mid a\right)\right) .
$$

The individual full rank condition is satisfied at the efficient point when vectors $p\left(a^{1}, a^{1}\right), \ldots, p\left(a^{H}, a^{1}\right)$ are linearly independent ${ }^{18}$. Note that this is satisfied in our prisoner's dilemma example.

Now let $d^{k} \equiv g_{1}\left(a^{k}, a^{1}\right)-g_{1}\left(a^{1}, a^{1}\right)$ be the gain from deviation to the $k$ th action at the efficient point. We first identify a transfer scheme $x_{1}(\omega)$ (in the Fudenberg-Levine algorithm) for player 1 which makes her indifferent among all actions. In particular, we find such a transfer $x_{1}(\omega)$ that $g_{1}\left(a^{k}, a^{1}\right)+\mathrm{E}\left[x_{1}(\omega) \mid a^{k}, a^{1}\right]$ is constant for all $k$. This provides an incentive for player 1 to choose any mixed action. The condition is given by

$$
\left(\begin{array}{c}
d^{2} \\
\vdots \\
d^{K}
\end{array}\right)=\left(\begin{array}{c}
p\left(a^{1}, a^{1}\right)-p\left(a^{2}, a^{1}\right) \\
\vdots \\
p\left(a^{1}, a^{1}\right)-p\left(a^{K}, a^{1}\right)
\end{array}\right)\left(\begin{array}{c}
x_{1}\left(\omega^{1}\right) \\
\vdots \\
x_{1}\left(\omega^{H}\right)
\end{array}\right),
$$

or,

$$
d=P\left(a^{1}\right) x_{1} .
$$

This condition states that the gain from any deviation (the left hand side) is exactly balanced with the loss in the expected transfer (the right hand side). Note that the argument of matrix $P(\cdot), a^{1}$, denotes the opponent's action that is fixed. Now we fix a base information structure $\bar{P}(\cdot)$ and assume that the public signal becomes insensitive to actions at the efficient point. To this end, we consider a family of distributions parametrized by $\xi$,

$$
P_{\xi}\left(a^{1}\right)=\xi \bar{P}\left(a^{1}\right)
$$

\footnotetext{
${ }^{16}$ We denote by $(1-\varepsilon) \circ a^{1}+\varepsilon \circ a^{m}$ a mixed action which plays $a^{1}$ and $a^{m}$ with probabilities $(1-\varepsilon)$ and $\varepsilon$ respectively.

${ }^{17}$ This result does not guarantee that an almost efficient payoff is sustained by a PPE. We also need to show that asymmetric action profiles can be supported without sacrificing much efficiency. However such detail is omitted.

${ }^{18}$ To satisfy this condition, we need $K \leq H$. We only stated the condition for player 1 , but by our symmetry assumption the same condition holds for player 2 .
} 
and let $\xi \rightarrow 0$, while holding the distributions elsewhere constant (i.e., $P_{\xi}\left(a^{k}\right)=\bar{P}\left(a^{k}\right)$ for $\left.k \neq 1\right)$. This embodies the idea that the signal becomes uninformative only at the efficient point $\left(a^{1}, a^{1}\right)$. Let $\bar{x}_{1}$ be a solution to $d=\bar{P}\left(a^{1}\right) x_{1}$, which exists because of the individual full rank condition (the rows of matrix $\bar{P}\left(a^{1}\right)$ are linearly independent). Clearly, $x_{1}=\frac{1}{\xi} \bar{x}_{1}$ is a solution to the incentive equation (3) for $P\left(a^{1}\right)=P_{\xi}\left(a^{1}\right)=\xi \bar{P}\left(a^{1}\right)$.

Our central assumption in this generalized example is that there is an action $a^{m}$ where $\left(p\left(a^{m}, a^{1}\right)-p\left(a^{m}, a^{k}\right)\right) \bar{x}_{1}<0$ for all $k=2, \ldots K$. That is, when player 1 is taking action $a^{m}$, any deviation by player 2 from the efficient action $a^{1}$ increases the expected value of the transfer $\bar{x}_{1}$ that we have constructed above (recall that $\bar{x}_{1}$ is a transfer which induces player 1 to choose any mixed action), In our prisoner's dilemma example, this condition is satisfied with $a^{m}=D$. In vector-matrix notation, our assumption is expressed as ${ }^{19}$

$$
\bar{P}\left(a^{m}\right) \bar{x}_{1} \ll 0
$$

Now we are ready to demonstrate that, for any fixed small number $\varepsilon>0$, a mixed action profile close to the efficient point, $\left((1-\varepsilon) \circ a^{1}+\varepsilon \circ a^{m}, a^{1}\right)$, is enforced by a transfer, when the signal becomes sufficiently uninformative at the efficient point (i.e., $\xi$ is sufficiently small). More precisely, for any given set of positive welfare weights $\lambda_{1}>0, \lambda_{2}>0$, we construct a transfer scheme $\left(x_{1}(\omega), x_{2}(\omega)\right)$ such that (i) $\lambda_{1} x_{1}(\omega)+\lambda_{2} x_{2}(\omega)=0$ for all $\omega$, and (ii) when each player's payoff is given by $g_{i}(a)+\mathrm{E}\left[x_{i}(\omega) \mid a\right]$, the profile $\left((1-\varepsilon) \circ a^{1}+\varepsilon \circ a^{m}, a^{1}\right)$ is a Nash equilibrium.

Let $x_{1}=\frac{1}{\xi} \bar{x}_{1}$. We have already seen that player 1 has an incentive to play any mixed action (and therefore to play the specified mixed action $\left.(1-\varepsilon) \circ a^{1}+\varepsilon \circ a^{m}\right)$. Define $x_{2}$ to satisfy $\lambda_{1} x_{1}+\lambda_{2} x_{2}=0$. Namely, let $x_{2}=-\frac{\lambda_{1}}{\lambda_{2} \xi} \bar{x}_{1}$. Now consider how much player 2 can gain in the stage game when she deviates from $a^{1}$ to $a^{k}$ (while player 1 is taking mixed action $\left.(1-\varepsilon) \circ a^{1}+\varepsilon \circ a^{m}\right)$. This is given by

$$
d_{2}^{k} \equiv g_{2}\left((1-\varepsilon) \circ a^{1}+\varepsilon \circ a^{m}, a^{k}\right)-g_{2}\left((1-\varepsilon) \circ a^{1}+\varepsilon \circ a^{m}, a^{1}\right),
$$

and we define a column vector of gains by $d_{2} \equiv\left(d_{2}^{2}, \ldots, d_{2}^{K}\right)^{\top}$. The equilibrium condition for player 2 specifies that those gains should be wiped out by the reduction of expected transfer, and in our notation it is expressed as

$$
d_{2} \leq P_{\xi}\left((1-\varepsilon) \circ a^{1}+\varepsilon \circ a^{m}\right) x_{2} .
$$

\footnotetext{
${ }^{19}$ Recall tha the $k$ th row of matrix $\bar{P}\left(a^{m}\right)$ is defined to be $p\left(a^{1}, a^{m}\right)-p\left(a^{k}, a^{m}\right)$, which is by our symmetry assumption equal to $p\left(a^{m}, a^{1}\right)-p\left(a^{m}, a^{k}\right)$.
} 
Note that the right hand side represents the reductions in the expected value of transfer $x_{2}$ which are associated with player 2's deviations, when player 1 is playing mixed action $(1-\varepsilon) \circ a^{1}+\varepsilon \circ a^{m}$. The right hand side of this inequality is equal to

$$
\begin{aligned}
& \left((1-\varepsilon) \xi \bar{P}\left(a^{1}\right)+\varepsilon \bar{P}\left(a^{m}\right)\right)\left(-\frac{\lambda_{1}}{\lambda_{2} \xi} \bar{x}_{1}\right) \\
= & -(1-\varepsilon) \frac{\lambda_{1}}{\lambda_{2}} \bar{P}\left(a^{1}\right) \bar{x}_{1}-\frac{\varepsilon}{\xi} \frac{\lambda_{1}}{\lambda_{2}} \bar{P}\left(a^{m}\right) \bar{x}_{1} .
\end{aligned}
$$

Under our assumption (4), the last term in the last line is a positive vector, and it diverges as $\xi \rightarrow 0$. In contrast, the first term in the last line is constant. Therefore, we conclude that the incentive condition for player 2 (inequality (5)) is satisfied for sufficiently small $\xi$.

Remark: We could weaken the crucial assumption (4) by

$$
\bar{P}\left(\alpha^{0}\right) \bar{x}_{1} \ll 0 .
$$

for some mixed action $\alpha^{0}$ whose support is contained in $\left\{a^{2}, \ldots, a^{K}\right\}$. Exactly the same proof goes through. Note that this condition is easier to satisfy than the original one.

\section{A Simple Example of the Folk Theorem without the Pairwise Full Rank Condition}

Besides providing the paradoxical result, our examples also point out a plausibility that efficiency in repeated games with imperfect monitoring is obtained under a much weaker conditions than is identified by the FLM folk theorem. In this section we elaborate on this issue, which is another point we would like to make with our examples.

The feasibility of efficient asymmetric punishment is the basic driving force of the FLM folk theorem, and one of the sufficient conditions to facilitate asymmetric punishment is the pairwise full rank condition. As we have seen in Sections 3 and 4, however, the preceding models have the property that the pairwise full rank condition is not satisfied but asymmetric punishment is feasible. This suggests that we may obtain the folk theorem under a weaker set of assumptions. In fact, a minor modification of our first example provides a fairly simple example where the folk theorem holds without the pairwise full rank condition. This is shown by an argument which closely follows our method of proofs in Section 3. 
Suppose that we have the same stage game payoff (the prisoners' dilemma) and assume that the signal $\omega=X, Y$ is distributed as follows.

$$
\begin{aligned}
P(X \mid C C) & =P(X \mid D D)=\frac{1}{2} \\
P(X \mid D C) & =\frac{1}{2}+\xi \\
P(X \mid C D) & =\frac{1}{2}-\xi
\end{aligned}
$$

It is not difficult to check that the FLM pairwise full rank condition is violated at any pure action profile because there are only two public signals. ${ }^{20}$ Nonetheless, the basic idea behind FLM is still valid. For example, consider $(C, C)$. It is possible to distinguish different players' deviations here; $X$ is more likely if player 1 deviates, and $Y$ is more likely if player 2 deviates (assuming that $\xi$ is positive). Therefore efficient punishments based on transfer of utility (á la FLM) can still be employed to support the efficient action profile without any efficiency loss.

The more tricky part is to support the asymmetric profiles with respect to a variety of hyperplanes. For example, consider another efficient point $(D, C)$. At this point, either player's deviation makes $Y$ more likely, so that asymmetric punishment is not feasible. However, since player 1's deviation (to $C$ ) is unprofitable, we may be able to transfer, without violating the incentive constraints, player 2's continuation payoff to player 1, when $Y$ is realized. We have to check if this transfer from 2 to 1 , which makes player 1 's deviation to $C$ more attractive, is not too large to wipe out the loss associated with the unprofitable deviation to $C$. It turns out that we can overcome this potential problem by the particular payoff structure of the Prisoners' dilemma (see Case 2 in the proof).

The enforceability of a given action profile on various hyperplanes is the core to achieve the folk theorem. FLM's pairwise full rank condition ensures this by requiring that the linear combinations of relevant signal distributions are distinct. Kandori and Matsushima [9] pointed out that this can be weakened by requiring that the convex combinations of relevant signal distributions are distinct. An essential condition of theirs is satisfied at $(C, C)$ in our example ${ }^{21}$. This fails at $(D, C)$ and $(C, D)$, however, as

\footnotetext{
${ }^{20}$ Indeed a weaker condition, pairwise identifiability condition ([7]), is violated at all the pure action profiles. On the other hand, the individual full rank condition is satisfied at every pure action profile.

${ }^{21}$ At this point, Player 1 , by deviating with a certain probablitiy, can create a convex combination of $\left(\frac{1}{2}, \frac{1}{2}\right)$ and $\left(\frac{1}{2}+\xi, \frac{1}{2}-\xi\right)$ (those vectors represent (probability of $X$, proba-
} 
either player's deviation creates the same distribution. Still, those points can be enforced on hyperplanes as we argued above, because of the special payoff structure. This suggests that we can obtain even a weaker set of conditions for the enforceability on hyperplanes, by imposing restrictions jointly on the information structure and the deviation payoffs. This issue is being addressed in work in preparation by Harrison Cheng [4].

Now we are ready to prove the following result.

Theorem 2 The model in this section violates the pairwise full rank condition but the folk theorem holds; for any $\xi>0$, $\lim _{\delta \rightarrow 1} E(\delta)=V^{*}$

Proof: We continue to use the Fudenberg-Levine Algorithm and demonstrate $Q(\xi)\left(=\lim _{\delta \rightarrow 1} E(\delta)\right)=V^{*}$.

Case 1, $\lambda_{1}, \lambda_{2} \leq 0$ and $\lambda \neq 0:(0,0) \in H(\lambda)$.

This case is obvious.

Case 2, $\lambda_{1}>0, \frac{\lambda_{2}}{\lambda_{1}} \leq \frac{d}{1+h}$ : $g(D, C) \in H(\lambda)$ (For such $\lambda, \lambda \cdot g(a)$ is maximized by $(D, C)$ )

This is established by showing that $(D, C)$ is enforceable while $\lambda \cdot x(\omega)=$ 0 is satisfied. The incentive constraints are

$$
\begin{aligned}
& g_{1}(D, C)+E\left[x_{1}(\omega) \mid D, C\right] \geq g_{1}(C, C)+E\left[x_{1}(\omega) \mid C, C\right] \\
& g_{2}(D, C)+E\left[x_{2}(\omega) \mid D, C\right] \geq g_{2}(D, D)+E\left[x_{2}(\omega) \mid D, D\right]
\end{aligned}
$$

which reduce to

$$
\begin{aligned}
& d \geq \xi\left(x_{1}(Y)-x_{1}(X)\right) \\
& h \leq \xi\left(x_{2}(X)-x_{2}(Y)\right) .
\end{aligned}
$$

The equality $\lambda \cdot x(\omega)=0$ can be satisfied by setting $x_{1}(Y)=x_{2}(Y)=0$, $x_{1}(X)=-\frac{\lambda_{2}}{\lambda_{1}} x_{2}(X)$. Then these two inequality constraints become

$$
\begin{aligned}
& d \geq \xi \frac{\lambda_{2}}{\lambda_{1}} x_{2}(X) \\
& h \leq \xi x_{2}(X) .
\end{aligned}
$$

biity of $Y)$ ). This is distinct from what Player 2's deviations create (convex combinations of $\left(\frac{1}{2}, \frac{1}{2}\right)$ and $\left.\left(\frac{1}{2}-\xi, \frac{1}{2}+\xi\right)\right)$, even though $\left(\frac{1}{2}, \frac{1}{2}\right),\left(\frac{1}{2}+\xi, \frac{1}{2}-\xi\right)$, and $\left(\frac{1}{2}-\xi, \frac{1}{2}+\xi\right)$ are linearly dependent (so that the pairwise full rank condition fails). 
If $\lambda_{2} \leq 0$, these constraints can be satisfied by taking $x_{2}(X)$ large enough. So suppose not. Then we can find a $x_{2}(X)$ to satisfy these inequalities if and only if $\frac{\lambda_{2}}{\lambda_{1}} h \leq d$, which is always satisfied because $\frac{\lambda_{2}}{\lambda_{1}} \leq \frac{d}{1+h}$ by assumption.

Case 3, $\lambda_{1}, \lambda_{2}>0: g(C, C) \in H(\lambda)$.

The incentive constraints are

$$
\begin{aligned}
d & \leq \xi\left(x_{1}(Y)-x_{1}(X)\right) \\
d & \leq \xi\left(x_{2}(X)-x_{2}(Y)\right) .
\end{aligned}
$$

These can be satisfied by, for example, $x_{1}(Y)=x_{2}(Y)=0, x_{1}(X)=$ $-\frac{\lambda_{2}}{\lambda_{1}} x_{2}(X)$ by taking $x_{2}(X)$ large enough while $\lambda \cdot x(\omega)=0$ is satisfied.

Note that the symmetric argument of Case 2 applies to the remaining case: $\lambda_{2}>0, \frac{\lambda_{1}}{\lambda_{2}} \leq \frac{d}{1+h}$. For this case, the incentive constraints are

$$
\begin{aligned}
& h \leq \xi\left(x_{1}(Y)-x_{1}(X)\right) \\
& d \geq \xi\left(x_{2}(X)-x_{2}(Y)\right) .
\end{aligned}
$$

Then we can set $x_{1}(X)=x_{2}(X)=0$ and $x_{2}(Y)=-\frac{\lambda_{1}}{\lambda_{2}} x_{1}(Y)$ and show that $g(C, D) \in H(\lambda)$ for such $\lambda$. Combining all these cases, it is clear that we have $Q(\xi)=V^{*}$. Q.E.D.

\section{Relationship to the Blackwell-Monotonicity}

The example in Section 3 (and Section 4) seemingly contradicts Kandori [8], which shows that the equilibrium payoff set becomes smaller when observability is reduced in Blackwell's sense. In this section we explain the precise relationship between our example and Kandori [8].

Recall that a signal $\omega^{\prime}$ is less informative than $\omega$ in Blackwell's sense, if there exists a function $q(\cdot \mid \cdot) \geq 0$ such that $\sum_{\omega^{\prime} \in \Omega} q\left(\omega^{\prime} \mid \omega\right)=1$ and $p^{\prime}\left(\omega^{\prime} \mid a\right)=\sum_{\omega \in \Omega} q\left(\omega^{\prime} \mid \omega\right) p(\omega \mid a)$ for each $\omega \in \Omega$ (where $p^{\prime}\left(\omega^{\prime} \mid a\right)$ and $p(\omega \mid a)$ denote the associated distribution functions). When this condition holds, we say that $\omega^{\prime}$ is a garbling of $\omega$. It is easy to see that, when public randomization device is available, any equilibrium strategy under the less informative signal $\omega^{\prime}$ can be mimicked under the better signal $\omega$. This is because the players can garble the signal by themselves via public randomization device. Inspection of the equilibrium conditions immediately shows 
that the strategy profile thus constructed is also an equilibrium under the better signal $\omega$. Hence, when $\omega^{\prime}$ is a garbling of $\omega$, we have $E_{\omega^{\prime}}(\delta) \subseteq E_{\omega}(\delta)$ for any $\delta \in[0,1)$, where $E_{\omega^{\prime}}(\delta)$ and $E_{\omega}(\delta)$ denote the public perfect equilibrium payoff sets under signal $\omega^{\prime}$ and $\omega$. Kandori's results [8] are built on this observation. Kandori pointed out that the same is true without public randomization when the signal is a continuous variable, and he went on to show $E_{\omega^{\prime}}(\delta) \subseteq E_{\omega}(\delta)$ under certain regularity conditions.

To demonstrate the precise relationship between those observations and our example, we now show that a similar result is obtained (without public randomization) in the current setting (i.e., with the finite signal space) in the limit $(\delta \rightarrow 1)$. Let $Q_{\omega^{\prime}}$ and $Q_{\omega}$ be the limit equilibrium payoff set $Q$ $\left(=\lim _{\delta \rightarrow 1} E(\delta)\right)$ associated with $\omega^{\prime}$ and $\omega$ respectively.

Proposition 1 If $\omega^{\prime}$ is a garbling of $\omega, Q_{\omega^{\prime}} \subseteq Q_{\omega}$.

Proof: Take any feasible $\left(\alpha^{\prime}, x^{\prime}\right)$ for the optimization problem with $p^{\prime}$ for direction $\lambda$. Consider $\left(\alpha^{\prime}, x\right)$ where $x$ is defined by $x(\omega)=\sum_{\omega^{\prime} \in \Omega} q\left(\omega^{\prime} \mid \omega\right) x^{\prime}\left(\omega^{\prime}\right)$. Then $\left(\alpha^{\prime}, x\right)$ is feasible for the optimization problem with $p$ for the direction $\lambda$ because the feasible set of $x$ is convex and the incentive constraints are automatically satisfied. Moreover, it is also clear that $\left(\alpha^{\prime}, x\right)$ achieves the same value as $\left(\alpha^{\prime}, x^{\prime}\right)$. This implies that $H_{p^{\prime}}(\lambda) \subset H_{p}(\lambda)$ for any $\lambda$, therefore $Q_{\omega^{\prime}} \subseteq Q_{\omega}$.Q.E.D.

Proposition 1 logically implies that, although our signalling structure gets "less informative" as $\xi \rightarrow 0$, it does not so in the sense of Blackwell. In fact, it is not so difficult to see that, in the limit, the assumption for Theorem $1\left(\lim _{\xi \rightarrow 0} \frac{\xi}{\Delta(\xi)}=0\right)$ precludes a signalling structure which is less informative by Blackwell's criterion.

Suppose that $p_{n+1}$ is a garbling of $p_{n}$ for $n=0,1, \ldots$ and $\left\|p_{n}(\cdot \mid C D)-p_{n}(\cdot \mid C C)\right\|$ converges to 0 as $n \rightarrow \infty$, where $p_{0}(\omega \mid a)=p(\omega \mid a)$. Since $p_{n+1}(\cdot \mid a)=$ $A_{n} p_{n}(\cdot \mid a)$ where $A_{n}$ is a matrix defined by

$$
A_{n}=\left(\begin{array}{ll}
q_{n}(X \mid X) & q_{n}(X \mid Y) \\
q_{n}(Y \mid X) & q_{n}(Y \mid Y)
\end{array}\right)
$$

for some $q_{n}(\cdot \mid \cdot)$, we have

$$
p_{n+1}(\cdot \mid a)=A_{n} \ldots A_{1} A_{0} p(\cdot \mid a)
$$

Let $A^{n}=A_{n} \ldots A_{1} A_{0}$. Since $\lim _{n \rightarrow \infty}\left\|A^{n}\left(p_{1}(\cdot \mid C D)-p_{1}(\cdot \mid C C)\right)\right\|=0$, it must be the case that

$$
\lim _{n \rightarrow \infty} A^{n}=\left(\begin{array}{cc}
x & x \\
1-x & 1-x
\end{array}\right)
$$


for some $x \in[0,1]$ as long as $\left\|p_{1}(\cdot \mid C D)-p_{1}(\cdot \mid C C)\right\|>0$. This implies that $\left\|p_{n}(\cdot \mid D D)-p_{n}(\cdot \mid D C)\right\|$ also converges to 0 , and, moreover, $\left\|p_{n}(\cdot \mid C D)-p_{n}(\cdot \mid C C)\right\|$ and $\left\|p_{n}(\cdot \mid D D)-p_{n}(\cdot \mid D C)\right\|$ converge to 0 at the same speed. ${ }^{22}$ This is in conflict with our assumption $\lim _{\xi \rightarrow 0} \frac{\xi}{\Delta(\xi)}=0$.

\section{References}

[1] D. Abreu, D. Pearce, and E. Stacchetti. Toward a theory of discounted repeated games with imperfect monitoring. Econometrica, 58:10411063, 1990.

[2] V. Bhaskar and I. Obara. Belief-based equilibria in the prisoners' dilemma with private monitoring. Journal of Economic Theory, 102:16$39,2002$.

[3] D. Blackwell and M. A. Girshick. Theory of Games and Statistical Decisions. John Wiley and Sons, New York, 1954.

[4] H. Cheng. Effciency and the no surplus condition in repeated games with imperfect monitoring. 2001. mimeo.

[5] J. C. Ely and J. Välimäki. A robust folk theorem for the prisoner's dilemma. Journal of Economic Theory, 102(1):84-105, 2002.

[6] D. Fudenberg and D. Levine. Efficiency and observability with long -run and short-run players. Journal of Economic Theory, 62:103-135, 1994.

[7] D. Fudenberg, D. K. Levine, and E. Maskin. The folk theorem with imperfect public information. Econometrica, 62:997-1040, 1994.

\footnotetext{
${ }^{22}$ Formally, let $\left(x_{1}, x_{2}\right)$ and $\left(y_{1}, y_{2}\right)$ be any two distinct signal distributions on $\{X, Y\}$ and $x=\left(x_{1}, x_{2}\right)^{\top}, y=\left(y_{1}, y_{2}\right)^{\top}$. Then

$$
\left\|A^{n}(x-y)\right\|=\left\|\left(\begin{array}{cc}
\mu_{n}^{\prime} & \mu_{n}^{\prime \prime} \\
1-\mu_{n}^{\prime} & 1-\mu_{n}^{\prime \prime}
\end{array}\right)(x-y)\right\|
$$

This is 0 for any $x, y$ if $\mu_{n}^{\prime}=\mu_{n}^{\prime \prime}$. So suppose not. Since $x_{1}-y_{1}=-\left(x_{2}-y_{2}\right)$,

$$
\left\|A^{n}(x-y)\right\|=\left|\mu_{n}^{\prime}-\mu_{n}^{\prime \prime}\right|\|x-y\|
$$

Therefore,

$$
\frac{\left\|A^{n}(p(\cdot \mid C C)-p(\cdot \mid C D))\right\|}{\left\|A^{n}(p(\cdot \mid D C)-p(\cdot \mid D D))\right\|}=\frac{\|p(\cdot \mid C C)-p(\cdot \mid C D)\|}{\|p(\cdot \mid D C)-p(\cdot \mid D D)\|} .
$$
}

That is, the ratio remains a constant along the sequence. 
[8] M. Kandori. The use of information in repeated games with imperfect monitoring. Review of Economic Studies, 59:581-594, 1992.

[9] M. Kandori and H. Matsushima. Private observation, communication and collusion. Econometrica, 66:627-652, 1998.

[10] M. Kandori and I. Obara. Efficiency in repeated games revisited: The role of private strategies. Econometrica, 74(2):499-519, 2006.

[11] E. Lehrer. Internal correlation in repeated games. International Journal of Game Theory, 19:431-456, 1991.

[12] E. Lehrer. On the equilibrium payoffs set of two player repeated games with imperfect monitoring. International Journal of Game Theory, 20:211-226, 1992.

[13] M. Piccione. The repeated prisoner's dilemma with imperfect private monitoring. Journal of Economic Theory, 102(1):70-83, 2002.

[14] T. Sekiguchi. Efficiency in repeated prisoner's dilemma with private monitoring. Journal of Economic Theory, 76:345-361, 1997. 